\title{
Changes in quality of life and dyspnoea after hospitalization in COVID-19 patients discharged at home
}

\author{
Pierachille Santus, ${ }^{1,2}$ Francesco Tursi, ${ }^{3}$ Giuseppe Croce, ${ }^{1,2}$ Chiara Di Simone,,${ }^{1,2}$ Francesca Frassanito, ${ }^{1,2}$ \\ Paolo Gaboardi, ${ }^{1,2}$ Andrea Airoldi, ${ }^{1}$ Marica Pecis, ${ }^{1}$ Giangiuseppe Negretto, ${ }^{1}$ Dejan Radovanovic ${ }^{1}$ \\ ${ }^{1}$ Division of Respiratory Diseases, Ospedale L. Sacco, ASST Fatebenefratelli-Sacco, Milan \\ ${ }^{2}$ Department of Biomedical and Clinical Sciences (DIBIC), Università degli Studi di Milano \\ ${ }^{3}$ Azienda Socio Sanitaria Territoriale di Lodi, Dipartimento Medico, USC Pneumologia, USS Servizio di Pneumologia, \\ Ospedale di Codogno, Lodi, Italy
}

\begin{abstract}
Background: To date, the effects of COVID-19 pneumonia on health-related quality of life (HRQoL) and dyspnoea are unknown.

Methods: In a real-life observational study, 20 patients with COVID-19-related pneumonia received usual care plus erdosteine (300 mg twice daily) for 15 days after hospital discharge following local standard operating procedures. At discharge (T0) and on Day 15 (T1), participants completed the St George's Respiratory Questionnaire (SGRQ), the modified Medical Research Council (mMRC) scale of dyspnoea during daily activity, the BORG scale for dyspnoea during exertion, and Visual Analogue Scale (VAS) for dyspnoea at rest. Paired t-tests compared scores at T0 and T1. Results: The mean (SD) SGRQ total score decreased from 25.5 (15.5) at T0 to $16.9(13.2)$ at $\mathrm{T} 1$ (p<0.01); 65\% of patients achieved a clinically important change of $\geq 4$ points. SGRQ domain scores (symptoms, activity, and impact) were also significantly reduced (all $\mathrm{p}<0.01$ ). The mean (SD) VAS score decreased from $1.6(1.7)$ to $1.4(2.5) ; \mathrm{p}<0.01$. The mean mMRC score decreased significantly $(\mathrm{p}=0.031)$ and $30 \%$ of patients achieved a clinically important change of $\geq 1$ point. The mean (SD) Borg score increased from 12.8 (4.2) to 14.3 (2.4); $\mathrm{p}<0.01$.

Conclusion: The present proof of concept study is the first to report HRQoL in patients with COVID-19. During 15 days after hospital discharge, patients reported significant improvements in HRQoL and dyspnoea at rest and during daily activities.
\end{abstract}

Key words: Antioxidant; coronavirus; COVID-19; dyspnea; erdosteine; quality of life.

Correspondence: Prof. Pierachille Santus, Department of Biomedical and Clinical Sciences (DIBIC), Division of Respiratory Diseases, Università degli Studi di Milano, Ospedale L. Sacco, ASST Fatebenefratelli-Sacco, Via G.B. Grassi 74, 20157 Milano, Italy.

Tel. +39.02.39042801 - Fax: +39.02.39042473.E-mail: pierachille.santus@unimi.it

Contributions: All authors contributed to the study conception and design. Material preparation, data collection and analysis were performed by PS, DR, FT, GC, CDS, FF, PG, AA, MP and GN. The first draft of the manuscript was written by PS and DR, and all authors commented on previous versions of the manuscript. All authors read and approved the final manuscript. PS had full access to all the data in the study and take responsibility for the integrity of the data and the accuracy of the data analysis and had final responsibility for the decision to submit for publication

Conflict of interest: Prof. Pierachille Santus reports research grants from Chiesi Farmaceutici, AirLiquide, Pfizer, Almirall, AstraZeneca, Edmond Pharma and Boehringer Ingelheim; personal fees from AstraZeneca, Boehringer Ingelheim, Edmond Pharma, Novartis, Menarini International, Guidotti, Mundipharma, Valeas, Berlin-Chemie and Zambon. Dr. Dejan Radovanovic reports personal fees from Astra Zeneca, Boehringer Ingelheim and Neopharmed Gentili. Drs. Francesco Tursi, Giuseppe Croce, Chiara Di Simone, Francesca Frassanito, Paolo Gaboardi, Andrea Airoldi, Marica Pecis and Giangiuseppe Negretto have no conflicts of interest to report.

Funding: The study was part of the project on "Respiratory Diseases in patients with COVID-19 patients" funded by the Department of Biomedical and Clinical Sciences "L. Sacco" of the University of Milan.

Availability of data and materials: The datasets used and/or analysed during the current study are available from the corresponding author on reasonable request.

Ethics approval and consent to participate: Ethical Committee approval: Comitato Etico Milano Area I; 17263/2020. Written informed consent was obtained from all patients.

Trial registration: Part of the prospective observational study registered at ClinicalTrials.gov: NCT04307459. 


\section{Introduction}

Since the start of the Coronavirus disease 2019 (COVID-19) pandemic due to infection with Severe Acute Respiratory Syndrome Coronavirus 2 (SARS-CoV-2), relatively little attention has been paid to the health-related quality of life (HRQoL) of patients with COVID-19 following discharge from hospital. Discharged COVID-19 patients who had severe disease (characterised by moderate to severe pneumonia) may experience ongoing/recurrent symptoms (including dyspnoea, cough, asthenia, fatigue) $[1,2]$, persistent impairment of lung function and exercise capacity [3], and psychological or psychosocial problems, which could impact their daily functioning and HRQoL in both the shortand long-term [4]. Dyspnoea, the subjective experience of breathing discomfort, typically develops 7 or 8 days after the onset of symptoms of COVID-19 and is prevalent in patients with severe disease [2,5-8]. Dyspnoea is the only symptom predictive of severe COVID-19 and intensive care unit (ICU) admission [9]. After hospital discharge, patients with severe COVID-19 may continue to experience dyspnoea both at rest and during exercise or daily activities. To aid recovery/rehabilitation, it is important to assess how much breathlessness impacts a patient's functioning and HRQoL and implement appropriate interventions/treatment [10].

The aim of this real-life study was to assess the HRQoL and symptoms (especially dyspnoea) of COVID-19 patients during the first 15 days after discharge home from hospital.

\section{Methods}

\section{Study design}

This was an observational, single-centre, open-label, real-world, proof of concept study of patients with COVID-19 following their discharge from the Respiratory High-Dependency Care Unit (RHDU) of Luigi Sacco University Hospital, Milan, Italy, after treatment for COVID-19-related pneumonia. The study was conducted between 1 April 2020 and 30 June 2020. While hospitalised in the RHDU, all participants had taken part in an observational study (NCT04307459; Comitato Etico Milano Area I; 17263/2020) for COVID-19-related pneumonia and severe respiratory failure, where they received standard care represented by oxygen supplementation and continuous positive airway pressure (CPAP) applied according to a management algorithm previously described [11]. Unless contraindicated, patients received hydroxychloroquine, lopinavir/ritonavir, antibiotics, xanthines, enteral nutrition and hydration, following local standard protocols and the Italian Society of Infectious and Tropical Diseases (SIMIT) recommendations [12,13]. Intravenous methylprednisolone was used in critically ill patients and patients with severe pneumonia at a maximum dose of $1 \mathrm{mg} / \mathrm{kg}$ per day in accordance with expert consensus statements $[13,14]$ and following the American Thoracic Society and Infectious Diseases Society of America guidelines [15]. Immunomodulation with off-label tocilizumab, a recombinant humanised anti-human interleukin (IL)6 receptor monoclonal antibody, at a dosage of $8 \mathrm{mg} / \mathrm{kg}$ body weight was administered in patients with signs of hyper-inflammatory syndrome and elevated levels of IL-6 [12]. Patients also received prophylactic low molecular weight heparin (LMWH) or were switched to a therapeutic dose of LMWH if already on chronic anticoagulant therapy or had signs of deep vein thrombosis or pulmonary embolism, or presented with D-dimer values $>5,000 \mathrm{mg} / \mathrm{L}$ fibrinogen equivalent units (FEU). After discharge from hospital, all participants received usual care and add-on erdosteine $300 \mathrm{mg}$ capsules twice daily for 15 days.

\section{Patients}

Participants of the inpatient observational study with COVID19-related pneumonia and severe respiratory failure (NCT04307459) were eligible for this real-life study if they were discharged from hospital to home. To be eligible for hospital discharge, all patients were required to have two negative SARS-CoV2 reverse transcription-polymerase chain reaction (RT-PCR) tests of nasopharyngeal swabs within a 48 -h period. Patients were excluded from the study if they had clinically significant or unstable concurrent disease that necessitated discharge from hospital to a rehabilitation unit, nursing home or long-term health care facility.

\section{Data collection and questionnaires}

Patient demographic and disease characteristics were collected at hospital admission and other clinical features/parameters, laboratory and imaging findings, and treatments used during hospitalisation were recorded.

Four questionnaires were completed by participants at the time of hospital discharge (T0) and on Day 15 post-discharge (T1): the St George's Respiratory Questionnaire (SGRQ), the modified Medical Research Council (mMRC) scale of dyspnoea during daily activity, the Borg Rating of Perceived Exertion (RPE) for dyspnoea during exertion, and a Visual Analogue Scale (VAS) for dyspnoea at rest. All questionnaires were self-administered and in the Italian language. Follow up assessment with the clinician was performed at $\mathrm{T} 1$ by telephone call.

The SGRQ is a pulmonary-specific HRQoL questionnaire that is divided into three domains measuring symptoms (including dyspnoea), activity limitation, and the social and emotional impact of disease [16]. Patients consider the last 15 days when rating these domains. The score range for each domain and the total score is from 0 (no impairment/ no effect on quality of life) to 100 (maximum impairment/ maximum perceived distress); thus, a higher score represents greater impairment or a poorer HRQoL. A 4-point change in the SGRQ total score is considered a clinically meaningful difference or a minimal clinically important difference (MCID) [17]. The mMRC dyspnoea scale grades the impact of dyspnoea on daily activities over the last 7 days, thereby quantifying the disability or physical limitations associated with dyspnoea $[18,19]$. It is a 5-point scale with scores ranging from $0-4$, where $0=I$ only get breathless with strenuous exercise; 1 = I get short of breath when hurrying on the level or up a slight hill; $2=$ I walk slower than people of the same age on the level because of breathlessness or I have to stop for breath when walking at my own pace on the level; $3=$ I stop for breath after walking 100 meters or after a few minutes on the level; 4 =I am too breathless to leave the house or I am breathless when dressing [20]. A 1-point change in the mMRC scale has been used to represent a clinically significant change [21].

The Borg RPE scale is a categorical scale that consists of descriptors linked to numbers where respondents rate their perceived level of exertion/physical activity and associated dyspnoea for the last time they performed an exercise of moderate intensity (e.g. climbing stairs or walking uphill). The scores range from 6 (no exertion) to 20 (maximal exertion) [20,22].

The dyspnoea VAS was a $10 \mathrm{~cm}$ horizontal line with anchors at each end and the descriptors 0 (no dyspnoea) and 10 (max dyspnoea), and a mark at 5 (in the middle of the VAS scale). Patients were asked to rate their dyspnoea at rest at the time of the assessment by putting a cross on the horizontal line.

\section{Statistical analysis}

The data are presented as mean \pm standard deviation (SD) or $n$ $(\%)$. Paired $t$-tests were used to compare scores at T0 and T1. The n (\%) patients who achieved a $\geq 4$-point reduction in the SGRQ 
total score and $\geq 1$-point reduction in the mMRC dyspnoea score was determined; these patients were considered to have achieved the MCID. For all tests, $p<0.05$ was considered statistically significant. Statistical analyses were performed using IBM SPSS Statistics for Windows, ver. 23.0. Armonk, NY.

\section{Results}

\section{Patient characteristics}

The study included 20 consecutive patients hospitalised in the RHDU for COVID-19-related pneumonia and severe respiratory failure who recovered and were discharged home. All 20 patients had two negative RT-PCR tests for SARS-CoV-2 from nasopharyngeal swabs prior to hospital discharge and were not required to be in quarantine for 14 days at home. The demographic and clinical characteristics of the patients at hospital admission are summarised in Table 1 . They had a mean age of 55 years and $85 \%$ were men. All patients had confirmed COVID-19 (positive swab test for SARS-CoV-2) and 60\% had comorbid cardiovascular disease, most frequently hypertension (40\%), and four patients (20\%) had chronic respiratory disease (COPD/asthma). The mean (SD) $\mathrm{PaO}_{2} / \mathrm{FiO}_{2}$ ratio at admittance was 283 (106) $\mathrm{mmHg}$, and 9 patients $(45 \%)$ had moderate/severe respiratory failure. Out of the 20 patients, 75\% had severe COVID-19 according to the WHO interim guidance [23]. Mean duration of hospitalisation was 17.7 days.

During hospitalisation, patients received conventional $\mathrm{O}_{2}$ therapy $(70 \%)$ and/or helmet CPAP $(50 \%)$. Also, $65 \%$ of patients were treated with corticosteroids, $90 \%$ with LMWH, 70\% with antibiotics, $50 \%$ with hydroxychloroquine, $45 \%$ with lopinavir/ritonavir, and $15 \%$ with tocilizumab. After hospital discharge, patients discontinued all COVID-19 therapy received during hospitalisation, except for three patients who continued prophylactic LMWH due to high D-dimer values. At home, patients continued their usual therapy for chronic conditions (e.g., hypertension, COPD, etc.) in addition to erdosteine treatment.

\section{Quality of life and dyspnoea}

All 20 patients completed the four questionnaires at both $\mathrm{T} 0$ and T1 (i.e., no patients were lost to follow up) and the mean (SD) scores are summarised in Table 2. At hospital discharge, the mean SGRQ total score was 25.5 and the mean scores for the domains of symptoms, activity and impact were $33.7,35.7$ and 17.3 , respectively. During the 15 days after hospital discharge, there was a significant reduction in SGRQ total score and in each of the three domain scores (symptoms, activity and impact). The Borg RPE mean score for dyspnoea on exertion was significantly lower at hospital discharge (1.28) than at Day 15 (14.3), whereas the mean VAS score for dyspnoea at rest was significantly higher at hospital discharge (1.6) than at Day 15 (1.4). The mean mMRC score decreased significantly from 1.1 to 0.9 between $\mathrm{T} 0$ and $\mathrm{T} 1$, showing a reduction in the impact of dyspnoea on daily activities. When patients were stratified by mMRC level at hospital discharge and Day 15 (Figure 1), the number of patients at mMRC levels 1 and 4 decreased from $\mathrm{T} 0$ to $\mathrm{T} 1$, the number of patients at mMRC level 2 increased from $\mathrm{T} 0$ to $\mathrm{T} 1$ and there was no change in the number of patients at mMRC level 3 .

Figure 2 shows that $65 \%$ of patients achieved a clinically significant improvement in the SGRQ total score between hospital discharge and Day 15, and $30 \%$ of patients achieved a clinically significant improvement in the mMRC dyspnoea scale from $\mathrm{T} 0$ to T1.

\section{Discussion}

To the best of our knowledge, this real-life, proof of concept study is the first to report the HRQoL of patients with COVID-19.

Table 1. Demographics and clinical parameters of participants at hospital admission and in-hospital treatment. Data are presented as mean (SD) unless otherwise indicated.

\begin{tabular}{|c|c|}
\hline Characteristic & COVID-19 patients $(n=20)$ \\
\hline Age, years & $55(15)$ \\
\hline Males, n (\%) & $17(85.0)$ \\
\hline BMI, kg/m² & $24.1(5.2)$ \\
\hline Current or ex-smoker, n (\%) & $8(40)$ \\
\hline SARS-CoV-2 positive swab, n (\%) & $20(100.0)$ \\
\hline Duration of hospitalisation, days & $17.7(11.5)$ \\
\hline $\begin{array}{l}\text { Gas exchange } \\
\text { Respiratory rate, breaths/min } \\
\mathrm{SpO}_{2}, \% \\
\mathrm{pH} \\
\mathrm{PaO}_{2}, \mathrm{mmHg} \\
\mathrm{PaCO}_{2} 2, \mathrm{mmHg} \\
\mathrm{PaO}_{2} / \mathrm{FiO}_{2}, \mathrm{mmHg}\end{array}$ & $\begin{array}{c}28(8) \\
91(3) \\
7.44(0.02) \\
87(37.2) \\
41(6.2) \\
283(106)\end{array}$ \\
\hline $\begin{array}{l}\mathrm{PaO}_{2} / \mathrm{FiO}_{2} \text { severity, } \mathrm{n}(\%) \\
\quad \leq 100, \mathrm{mmHg} \\
\quad 101-200, \mathrm{mmHg} \\
201-300, \mathrm{mmHg} \\
>300, \mathrm{mmHg}\end{array}$ & $\begin{array}{l}2(10) \\
2(10) \\
5(25) \\
11(55)\end{array}$ \\
\hline $\begin{array}{l}\text { Blood count and biochemistry } \\
\text { Haemoglobin, g/l } \\
\text { Platelets, per } 10^{9} / \mathrm{L} \\
\text { White blood cells, per } 10^{9} / \mathrm{L} \\
\text { Neutrophils, per } 10^{9} / \mathrm{L} \\
\text { Lymphocytes, per } 10^{9} / \mathrm{L} \\
\text { D-dimer, mg/L FEU } \\
\text { Troponin T, ng/l }\end{array}$ & $\begin{array}{l}12.8(1.8) \\
278(135) \\
9.3(5.9) \\
7.1(5.7) \\
1.4(0.7) \\
2887(4126) \\
62(158)\end{array}$ \\
\hline $\begin{array}{l}\text { Radiological pattern, n (\%) } \\
\text { Interstitial } \\
\text { Bilateral ground glass opacities } \\
\text { Parenchymal consolidations }\end{array}$ & $\begin{array}{l}20(100) \\
15(75) \\
9(45)\end{array}$ \\
\hline $\begin{array}{l}\text { Comorbidities, } \mathrm{n}(\%) \\
\text { Cardiovascular diseases } \\
\text { Any cardiovascular disease } \\
\text { Hypertension } \\
\text { Ischaemic heart disease } \\
\text { Heart failure }\end{array}$ & $\begin{array}{l}12(60) \\
8(40) \\
3(15) \\
1(5)\end{array}$ \\
\hline $\begin{array}{l}\text { Other } \\
\text { Asthma } \\
\text { Diabetes mellitus } \\
\text { COPD } \\
\text { Active solid cancer }\end{array}$ & $\begin{array}{l}3(15) \\
2(10) \\
1(5) \\
1(5)\end{array}$ \\
\hline $\begin{array}{l}\text { In-hospital treatment, n (\%) } \\
\text { LMWH } \\
\text { Antibiotics } \\
\text { O2 therapy } \\
\text { Corticosteroids } \\
\text { CPAP } \\
\text { Hydroxychloroquine } \\
\text { Lopinavir/ritonavir } \\
\text { Tocilizumab }\end{array}$ & $\begin{array}{l}18(90) \\
14(70) \\
14(70) \\
13(65) \\
10(50) \\
10(50) \\
9(45) \\
3(15)\end{array}$ \\
\hline
\end{tabular}

BMI, body mass index; COPD, chronic obstructive pulmonary disease; CPAP, continuous positive airway pressure; FEU, fibrinogen equivalent units; $\mathrm{LMWH}$, low molecular weight heparin; $\mathrm{SpO}_{2}$, peripheral oxygen saturation; $\mathrm{PaO}_{2}$, partial arterial pressure of oxygen; $\mathrm{PaCO}_{2}$, partial arterial pressure of carbon monoxide; $\mathrm{PaO}_{2} / \mathrm{FiO}_{2}$, ratio between $\mathrm{PaO}_{2}$ and fraction of inspired oxygen. 
We used a respiratory-specific instrument (SGRQ) to determine HRQoL after hospital discharge following treatment for COVID19-related pneumonia and severe respiratory failure. After 15 days from hospital discharge, patients reported a statistically and clinically significant improvement in their overall HRQoL, as shown by the increase in SGRQ total score. This was accompanied by significant improvements in all domains of the SGRQ (symptom, activity and impact). Also, for $65 \%$ of patients, the change in SGRQ total score exceeded the MCID of 4 points.

These findings are important because they show a rapid improvement in HRQoL in the first two weeks after hospital discharge among patients who had been treated in hospital for severe COVID-19 and who were still experiencing symptoms (especially dyspnoea) at the time of discharge. The mean SGRQ total score decreased from 25.5 points at hospital discharge to 16.9 points at Day 15. The latter score shows that patients still have an impaired HRQoL relative to a general population-based norm (8.41), but it is lower than the SGRG total scores reported for patients with asthma (males 23.15, females 21.68) and for patients with COPD (males 18.18, females 12.55) [24].

There are no published studies of HRQoL scores in COVID-19 patients to compare our findings with. However, a systematic review of the long-term ( $>3$ months) outcomes of patients who had been hospitalised with Severe Acute Respiratory Syndrome (SARS) or Middle East Respiratory Syndrome (MERS), showed that HRQoL (measured using the SF-36 generic instrument) was still considerably reduced at 6 months after hospital discharge compared with normal healthy subjects [25]. HRQoL was assessed using the SGRQ in a few studies of SARS survivors. In a 12-month prospective follow up of SARS patients after hospital discharge, the SGRG total score improved from a median of 24 points at 3 months to 17 points at 6 months and 18 points at 12 months [26]. Similarly, the mean SGRG total score for SARS survivors in another study was 15.1 points at 12 months follow up [27]. Survivors of acute respiratory distress syndrome (ARDS) also reported a reduced HRQoL [28], although few studies used the SGRQ and only assessed HRQoL several months after hospital discharge [29]. However, in a small observational study of nine patients who survived ARDS due to severe influenza A (H1N1) pneumonitis, the median SGRQ total score decreased from 29.4 points at 1 month post-discharge to 4.6 points at 6 months post-discharge [30]. It is difficult to make comparisons between survivors of ARDS (who are generally treated in an ICU and have a high mortality rate) and the non-ICU hospitalised COVID-19 patients in our study.

Dyspnoea is one of the major clinical features of COVID-19 and is common among patients with severe disease, as in the present study. Even after hospital discharge, patients can continue to experience dyspnoea and relief of breathlessness is an important aim of treatment/rehabilitation. As patients can modify their level of breathlessness by changing their degree of physical activity, it is important to assess dyspnoea at rest and during exercise or daily activities.

All 20 patients in our study had dyspnoea at the time they were discharged home from hospital. As they would have had limited movement during hospitalisation and likely became more active at home, the different measures of dyspnoea were used are particularly informative. Our results show that dyspnoea at rest (VAS) and the impact of dyspnoea on daily activities (mMRC) improved significantly during the 15 days following hospital discharge. Notably, three out of the four patients with a high level of dyspnoea at hospital discharge (mMRC level 4) were no longer at this level on day 15 . The reduction in the number of patients at mMRC level 1 on Day 15 and the increase in the number of patients at mMRC level 2 , probably reflects greater patient activity post-dis-
Table 2. Quality of life and dyspnoea scores at hospital discharge (T0) and at 15 days post-discharge (T1) for the 20 patients.

\begin{tabular}{lccc} 
& $\begin{array}{c}\text { At discharge (T0) } \\
\text { Mean (SD) }\end{array}$ & $\begin{array}{c}\text { At 15 days (T1) } \\
\text { Mean (SD) }\end{array}$ & p $^{*}$ \\
SGRQ $^{\circ}$ & & & \\
Symptoms (range 0-100) & $33.7(18.0)$ & $16.7(12.9)$ & $<0.01$ \\
Activity (range 0-100) & $35.7(24.2)$ & $28.3(23.3)$ & $<0.01$ \\
Impact (range 0-100) & $17.3(15.9)$ & $10.6(10.7)$ & $<0.01$ \\
Total score (range 0-100) & $25.5(15.5)$ & $16.9(13.2)$ & $<0.01$ \\
Borg RPE (range 6-20) & $12.8(4.2)$ & $14.3(2.4)$ & $<0.01$ \\
VAS $^{\S}$ (range 0-10) & $1.6(1.7)$ & $1.4(2.5)$ & $<0.01$ \\
mMRC $^{\wedge}$ (range 0-4) & $1.1(1.2)$ & $0.9(0.9)$ & 0.03 \\
\hline
\end{tabular}

${ }^{*}$ p-values for comparisons between T0 and T1 were calculated using paired t-tests; SGRQ, Saint George's Respiratory Questionnaire: ${ }^{\circ}$ lower score denotes better health-related quality of life; RPE, rate of perceived exertion: "lower score denotes lower level of exertion and associated dyspnoea; VAS, Visual Analogue Scale: \$lower score denotes less dyspnoea at rest; mMRC, modified Medical Research Council dyspnoea scale: ^lower score denotes less activity limitation due to dyspnoea.

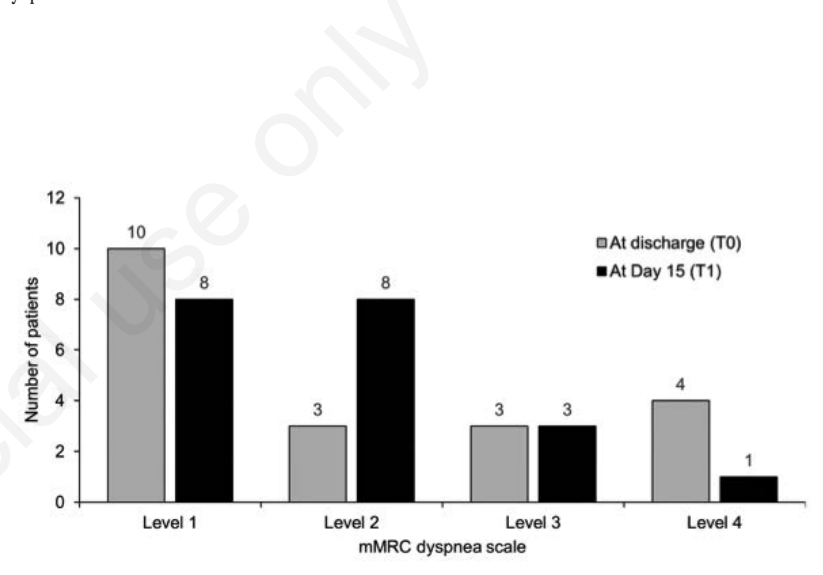

Figure 1. Changes in mMRC dyspnoea scale after discharge. Number of patients stratified by level on the mMRC dyspnoea scale at hospital discharge (T0) and 15 days post-discharge (T1). Level 1 = I get short of breath when hurrying on the level or up a slight hill; level 2 = I walk slower than people of the same age on the level because of breathlessness or I have to stop for breath when walking at my own pace on the level; level $3=$ I stop for breath after walking 100 meters or after a few minutes on the level; level $4=I$ am too breathless to leave the house or I am breathless when dressing.

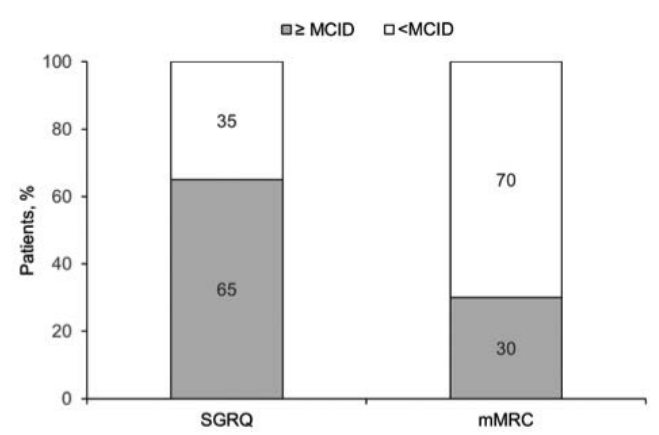

Figure 2. Proportion of patients that reached the MCID in SGRQ and $\mathrm{mMRC}$ after discharge. Percentage of patients $(n=20)$ who achieved a clinically important change in the SGRQ total score (MCID $\geq 4$ points) and in mMRC dyspnoea scale score (MCID $\geq 1$ point) between hospital discharge and Day 15. MCID minimal clinically important difference. 
charge. Similarly, the higher mean Borg RPE score on Day 15 compared with that at hospital discharge probably reflects the patients becoming more active during the two weeks after discharge, thereby increasing the score for dyspnoea on effort.

Like other coronavirus infections, SARS-CoV-2 infection of the respiratory tract may induce oxidative stress, which reflects an imbalance between the production of free radicals or ROS and the antioxidant defence system $[31,32]$. Some line of evidence currently suggests that excessive production of free radicals or ROS may lead to a dysregulated innate immune response, glutathione repletion, excessive inflammation and the cytokine storm seen in some patients with severe COVID-19 pneumonia [33]. The addition of erdosteine to usual therapy was driven by the hypothesis that antioxidant therapy might be beneficial in reducing the oxidative stress perpetrated by the viral infection by restoring the reduced glutathione in the respiratory system, thus promoting molecular and cellular repair [33].

The major strength of this study is that it is the first to assess HRQoL in patients following their discharge from hospital after treatment for moderate/severe COVID-19. Also, we used questionnaires that are widely used and well-validated, and there was no loss to follow up as all patients completed these questionnaires.

\section{Study limitations}

Our real-life, proof of concept study has several limitations. First, the sample size was small because not all COVID-19 patients in the RHDU were discharged home; approximately $50 \%$ of patients were discharged to other hospitals for rehabilitation. Also, some patients could not be enrolled in our study because they were homeless and unavailable for follow up post-discharge. Moreover, at the time the study was done, the number of patients in hospital with severe COVID-19 was declining and study enrolment stopped when the last patient in the RHDU was discharged home. Another limitation of our study is the lack of a comparison or control group, which limits the interpretation of our findings. In fact, the anti-oxidant erdosteine was added to the usual care in all patients at hospital discharge, so that any speculation on the independent effects of erdosteine on the improvements in quality of life should be taken with great caution. Indeed, a comparative, randomized trial would could be needed to verify the potential hypothesis that erdosteine can facilitate lung healing affecting quality of life or respiratory symptoms. Finally, the duration of the follow up period could have biased the results, limiting the observation to an initial curve of the post-discharge dynamics of symptoms and quality of life in patients with COVID-19.

\section{Conclusions}

Our real-life study is the first to report the objective assessment of HRQoL of patients with COVID-19. The results suggest that patients who had been treated in hospital for COVID-19-related pneumonia and severe respiratory failure had an impaired HRQoL at hospital discharge, which improved significantly during the first 15 days post-discharge. The improvements in overall HRQoL were clinically significant in approximately two-thirds of patients and were accompanied by significant improvements in the domains of symptoms, activities and impact. In addition, dyspnoea at rest and during daily activities improved during the 15 days post-discharge. To confirm our results, further studies are warranted to explore the long term effects and consequences of COVID-19 pneumonia on patients' quality of life and respiratory symptoms.

\section{Acknowledgements}

Medical writing support, under the direction of the authors, was provided by Deirdre Elmhirst, PhD, of Elmhirst Scientific Consultancy Ltd, funded by Edmond Pharma.

\section{List of abbreviations}

COPD: $\quad$ chronic obstructive pulmonary disease;

COVID-19: Coronavirus Induced Disease 2019;

CPAP: $\quad$ continuous positive airway pressure;

FEU: fibrinogen equivalent units;

GSH: glutathione;

HRQoL: health-related quality of life;

ICU: intensive care unit;

LMWH: $\quad$ low molecular weight heparin;

MCID: $\quad$ minimal clinically important difference;

mMRC: $\quad$ modified Medical Research Council;

NAC: $\quad$ N-acetylcysteine;

RHDU: Respiratory High-Dependency Care Unit;

ROS: $\quad$ reactive oxygen species;

RPE: $\quad$ Rating of Perceived Exertion;

SGRQ: $\quad$ St George's Respiratory Questionnaire;

SARS-CoV-2: Severe Acute Respiratory Syndrome

Coronavirus 2;

SIMIT: $\quad$ Italian Society of Infectious and

VAS: $\quad$ Visual Analogue Scale.

\section{References}

1. Ye G, Pan Z, Pan Y, Deng Q, Chen L, Li J, et al. Clinical characteristics of severe acute respiratory syndrome coronavirus 2 reactivation. J Infect 2020;80:e14-7. doi: 10.1016/j.jinf. 2020.03.001.

2. Zhou F, Yu T, Du R, Fan G, Liu Y, Liu Z, et al. Clinical course and risk factors for mortality of adult inpatients with COVID19 in Wuhan, China: a retrospective cohort study. Lancet 2020;395:1054-62. doi: 10.1016/S0140-6736(20)30566-3.

3. Mo X, Jian W, Su Z, Chen M, Peng H, Peng P, et al. Abnormal pulmonary function in COVID-19 patients at time of hospital discharge. Eur Respir J 2020;55:2001217. doi: 10.1183/1399 3003.01217-2020.

4. Zheng Z, Yao Z, Wu K, Zheng J. Patient follow-up after discharge after COVID-19 pneumonia: considerations for infectious control. J Med Virol 2020. https://doi.org/10.1002/ jmv.25994.

5. Guan WJ, Ni ZY, Hu Y, Liang WH, Ou CQ, He JX, et al. Clinical characteristics of coronavirus disease 2019 in China. N Engl J Med 2020;382:1708-20. doi: 10.1056/NEJMoa 2002032.

6. Huang C, Wang Y, Li X, Ren L, Zhao J, Hu Y, et al. Clinical features of patients infected with 2019 novel coronavirus in Wuhan, China. Lancet 2020;95:497-506. doi: 10.1016/S01406736(20)30183-5.

7. Liu L, Gao JY, et al. Clinical characteristics of 51 patients discharged from hospital with COVID-19 in Chongqing, China. MedRxiv 2020. Accessed: 29 June 2020. doi: 10.1101/2020. 02.20.20025536

8. Wang D, Yin Y, Hu C, Liu X, Zhang X, Zhou S, et al. Clinical course and outcome of 107 patients infected with the novel coronavirus, SARS-CoV-2, discharged from two hospitals in Wuhan, China. Crit Care 2020;24:188. doi: 10.1186/s13054020-02895-6. 
9. Jain V, Yuan JM. Predictive symptoms and comorbidities for severe COVID-19 and intensive care unit admission: a systematic review and meta-analysis. Int $J$ Public Health 2020;65:533-46. doi: 10.1007/s00038-020-01390-7.

10. Vitacca M, Lazzeri M, Guffanti E, Frigerio P, D’Abrosca F, Gianola $\mathrm{S}$, et al. Italian suggestions for pulmonary rehabilitation in COVID-19 patients recovering from acute respiratory failure: results of a Delphi process. Monaldi Arch Chest Dis 2020;90:1444. doi: 10.4081/monaldi.2020.1444

11. Radovanovic D, Rizzi M, Pini S, Saad M, Chiumello DA, Santus P. Helmet CPAP to treat acute hypoxemic respiratory failure in patients with COVID-19: A management strategy proposal. J Clin Med 2020;9:1191. doi: 10.3390/jcm9041191

12. Società Italiana di Malattie Infettive e Tropicali (SIMIT). Sezione Regione Lombardia. Vademecum per la cura delle persone con malattia da COVI-19. Ed. 2.0, 13 March 2020. Accessed: 6 August 2020. Available from: https://www. sicp.it/wp-content/uploads/2020/04/SIMIT_Vademecum-curapz-Covid19_13-mar-2020.pdf

13. Bassetti M, Giacobbe DR, Aliberti S, Barisione E, Centanni S, De Rosa FG, al. Balancing evidence and frontline experience in the early phases of the COVID-19 pandemic: current position of the Italian Society of Anti-infective Therapy (SITA) and the Italian Society of Pulmonology (SIP). Clin Microbiol Infect 2020;26:880-94. doi: 10.1016/j.cmi.2020.04.031

14. Shang L, Zhao J, Hu Y, Du R, Cao B. On the use of corticosteroids for 2019-nCoV pneumonia. Lancet 2020;395:683-4. doi: 10.1016/S0140-6736(20)30361-5

15. Metlay JP, Waterer GW, Long AC. Diagnosis and treatment of adults with community-acquired pneumonia. An official clinical practice guideline of the American Thoracic Society and Infectious Diseases Society of America. Am J Respir Crit Care Med 2019;200:e45-67. doi: 10.1164/rccm.201908-1581ST

16. Jones PW, Quirk FH, Baveystock CM, Littlejohns P. A selfcomplete measure of health status for chronic airflow limitation. The St George's Respiratory Questionnaire. Am Rev Respir Dis 1992;145:1321-7. doi: 10.1164/ajrccm/145.6.1321

17. Jones PW. St George's Respiratory Questionnaire: MCID. COPD 2005;2:75-9. doi: 10.1081/copd-200050513

18. Fletcher CM. Standardised questionnaire on respiratory symptoms: a statement prepared and approved by the MRC Committee on the Aetiology of Chronic Bronchitis (MRC breathlessness score). Br Med J 1960;2:1665.

19. Bestall JC, Paul EA, Garrod R, Garnham R, Jones PW, Wedzicha JA. Usefulness of the Medical Research Council (MRC) dyspnoea scale as a measure of disability in patients with chronic obstructive pulmonary disease. Thorax 1999;54:581-6.

20. Crisafulli E, Clini EM. Measures of dyspnea in pulmonary rehabilitation. Multidiscip Respir Med 2010;5:202-10. doi: 10.1186/2049-6958-5-3-202

21. de Torres JP, Pinto-Plata V, Ingenito E, Bagley P, Grey A, Berger R, et al. Power of outcome measurements to detect clinically significant changes in pulmonary rehabilitation of patients with COPD. Chest 2002;121:1092-8. doi: 10.1378/chest.121.4.1092

22. Borg GA. Psychophysical bases of perceived exertion. Med Sci Sports Exerc 1982;14:377-81.

23. WHO. Clinical management of severe acute respiratory infection (SARI) when COVID-19 disease is suspected. Accessed: 13 March 2020. Available from: https://www.who.int/publications-detail/clinical-managementof-severe-acute-respiratoryinfection-when-novel-coronavirus-(ncov)-infectionis-suspected.pdf

24. Ferrer M, Villasante C, Alonso J, Sobradillo V, Gabriel R, Vilagut $\mathrm{G}$, et al. Interpretation of quality of life scores from the St George's Respiratory Questionnaire. Eur Respir J 2002;19:405-13.

25. Ahmed H, Patel K, Greenwood DC, Halpin S, Lewthwaite P, Salawu A, et al. Long-term clinical outcomes in survivors of severe acute respiratory syndrome (SARS) and middle east respiratory syndrome (MERS) coronavirus outbreaks after hospitalisation or ICU admission: a systemic review and metaanalysis. J Rehab Med 2020;52:jrm00063. doi: 10.2340/ 16501977-2694.

26. Tansey CM, Louie M, Loeb M, Gold WL, Muller MP, de Jager $\mathrm{J}$, et al. One-year outcomes and health care utilisation in survivors of severe acute respiratory syndrome. Arch Int Med 2007;167:1312-20.

27. Ong KC, Wei-Keong A, Lee LS, Kaw G, Kwek SK, Leow MKS, et al. 1-Year pulmonary function and health status in survivors of severe acute respiratory syndrome. Chest 2005;128:1393-400.

28. Dowdy DW, Eid MP, Dennison CR, Mendez-Tellez PA, Herridge MS, Guallar E, et al. Quality of life after acute respiratory distress syndrome: a meta-analysis. Intensive Care Med 2006;32:1115-24. doi: 10.1007/s00134-006-0217-3

29. Davidson TA, Caldwell ES, Curtis JR, Hudson LD, Steinberg KP. Reduced quality of life in survivors of acute respiratory distress syndrome compared with critically ill control patients. JAMA 1999;281:354-60. doi: 10.1001/jama.281.4.354

30. Hsieh MJ, Lee WC, Cho HY, Wu MF, Hu HC, Kao KC, et al. Recovery of pulmonary functions, exercise capacity, and quality of life after pulmonary rehabilitation in survivors of ARDS due to severe influenza A (H1N1) pneumonitis. Influenza Other Respir Viruses 2018;12:643-8. doi: 10.1111/irv.12566

31. Delgado-Roche L, Mesta F. Oxidative stress as key player in Severe Acute Respiratory Syndrome Coronavirus (SARSCoV) infection. Arch Med Res 2020;51:384-7. doi: 10.1016/j.arcmed.2020.04.019

32. Polonikov A. Endogenous deficiency of glutathione as the most likely cause of serious manifestations and death in COVID-19 patients. ACS Infect Dis 2020;6:1558-62. doi: 10.1021/acsinfecdis.0c00288

33. Silvagno, Vernone A, Pescarmona GP. The role of glutathione in protecting against the severe inflammatory response triggered by COVID-19. Antioxidants (Basel) 2020;9:624. doi: 10.3390/antiox 9070624

Received for publication: 7 September 2020. Accepted for publication: 30 September 2020.

This work is licensed under a Creative Commons Attribution-NonCommercial 4.0 International License (CC BY-NC 4.0).

(C) Copyright: the Author(s), 2020

Licensee PAGEPress, Italy

Multidisciplinary Respiratory Medicine 2020; 15:713

doi:10.4081/mrm.2020.713 